\title{
Erratum to: Intact IFN- $\gamma$ R1 Expression and Function Distinguishes Langerhans Cell Histiocytosis From Mendelian Susceptibility to Mycobacterial Disease
}

\author{
Willemijn T. Quispel ${ }^{1}$ - Janine A. Stegehuis-Kamp ${ }^{1}$ - Susy J. Santos ${ }^{1}$. \\ Annelies van Wengen $^{2} \cdot$ Edward Dompeling $^{3} \cdot$ R. Maarten Egeler ${ }^{4,5}$. \\ Esther van de Vosse $^{2} \cdot$ Astrid G. S. van Halteren ${ }^{1}$
}

Published online: 19 January 2016

(C) Springer Science+Business Media New York 2016

Erratum to: J Clin Immunol (2014)

DOI 10.1007/s10875-013-9959-1

The original version of this article unfortunately contained mistakes in Table 1. The correct version is presented below.

The online version of the original article can be found at http://dx.doi.org/ 10.1007/s10875-013-9959-1.

\section{Astrid G. S. van Halteren}

a.g.s.van_Halteren@lumc.nl

1 Immunology Laboratory, Willem Alexander Children's Hospital, Leiden University Medical Center, Leiden, The Netherlands

2 Department of Infectious diseases, Leiden University Medical Center, Leiden, The Netherlands

3 Department of Paediatric Pulmonology, Maastricht University Medical Center, Maastricht, The Netherlands

4 Willem Alexander Children's Hospital, Leiden University Medical Center, Leiden, The Netherlands

5 Division of Hematology/Oncology, Hospital for Sick Children/ University of Toronto, Toronto, Canada 


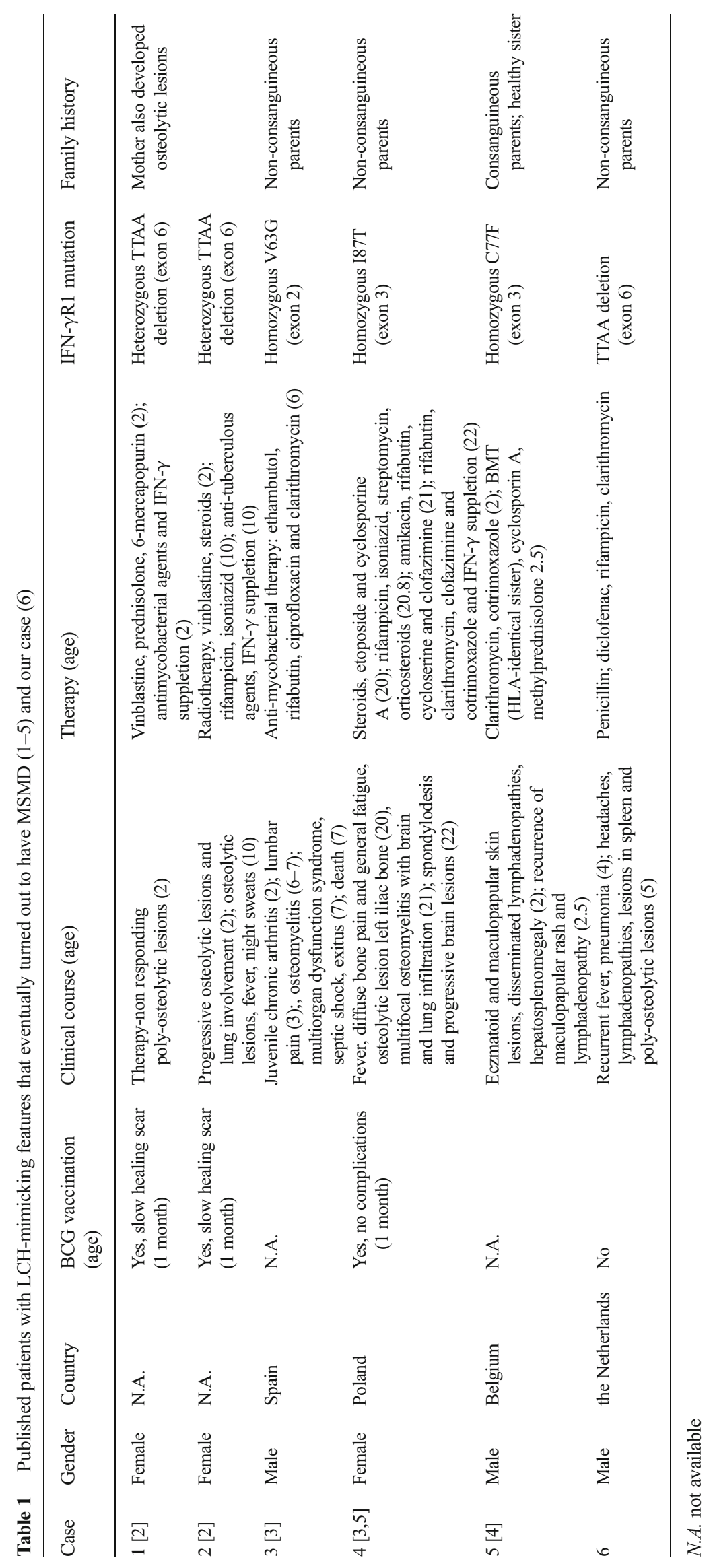

